\title{
Exposure of beta-tubulin regions defined by antibodies on an Arabidopsis thaliana microtubule protofilament model and in the cells
}

\author{
Yaroslav Blume ${ }^{1 \dagger}$, Alla Yemets $^{1 \dagger}$, Yarina Sheremet ${ }^{1}$, Alexey Nyporko ${ }^{1}$, Vadym Sulimenko ${ }^{2}$, Tetyana Sulimenko ${ }^{2}$, \\ Pavel Dráber ${ }^{2^{*}}$
}

\begin{abstract}
Background: The function of the cortical microtubules, composed of $\alpha \beta$-tubulin heterodimers, is linked to their organizational state which is subject to spatial and temporal modulation by environmental cues. The role of tubulin posttranslational modifications in these processes is largely unknown. Although antibodies against small tubulin regions represent useful tool for studying molecular configuration of microtubules, data on the exposure of tubulin epitopes on plant microtubules are still limited.

Results: Using homology modeling we have generated an Arabidopsis thaliana microtubule protofilament model that served for the prediction of surface exposure of five $\beta$-tubulin epitopes as well as tyrosine residues. Peptide scans newly disclosed the position of epitopes detected by antibodies 18D6 ( $\beta 1-10)$, TUB2.1 ( $\beta 426-435)$ and TU-14 ( $\beta 436-445)$. Experimental verification of the results by immunofluorescence microscopy revealed that the exposure of epitopes depended on the mode of fixation. Moreover, homology modeling showed that only tyrosines in the C-terminal region of $\beta$-tubulins (behind $\beta 425$ ) were exposed on the microtubule external side.

Immunofluorescence microscopy revealed tyrosine phosphorylation of microtubules in plant cells, implying that $\beta$-tubulins could be one of the targets for tyrosine kinases.
\end{abstract}

Conclusions: We predicted surface exposure of five $\beta$-tubulin epitopes, as well as tyrosine residues, on the surface of $A$. thaliana microtubule protofilament model, and validated the obtained results by immunofluorescence microscopy on cortical microtubules in cells.

The results suggest that prediction of epitope exposure on microtubules by means of homology modeling combined with site-directed antibodies can contribute to a better understanding of the interactions of plant microtubules with associated proteins.

\section{Background}

Microtubules are dynamic cytoskeletal polymers essential for various cell functions such as intracellular organization, ordered vesicle transport, cell division and establishment of cell polarity. In higher plants, several distinct microtubular arrays have been identified, namely the interphase cortical array, preprophase band, mitotic spindle and phragmoplast [1]. The basic building blocks of microtubules are heterodimers of globular $\alpha$ - and $\beta$-tubulin subunits. They are arranged in a head-to-tail

\footnotetext{
* Correspondence: paveldra@img.cas.cz

† Contributed equally

${ }^{2}$ Institute of Molecular Genetics, Academy of Sciences of the Czech Republic,
} 14220 Prague, Czech Republic fashion to form 13 protofilaments that constitute cylindrical microtubules with outer diameter around $25 \mathrm{~nm}$ [2]. In A. thaliana tubulin subunits are encoded by small gene families, six for $\alpha$-tubulin [3] and nine for $\beta$-tubulin [4]. It has been proposed that the function of microtubules is modulated by highly diverse posttranslational modifications of tubulin dimers [5].

A major advance step in understanding the microtubule function is marked by the solution of its structure, based on docking the high-resolution structure of brain tubulin, studied by electron crystallography [6,7], into lower-resolution microtubule maps imaged by electron cryomicroscopy [8-10]. The microtubular surface displays a surprisingly large number of binding sites, with 
numerous proteins binding to the outside surface and a multitude of small ligands binding to the inside of microtubules [11]. Some structural interactions with other molecules including nucleotides, drugs, microtubuleassociated proteins (MAPs) and motor proteins were predicted [2]. Microtubule models also provide the opportunity to predict the surface location of small antibody epitopes, as well as posttranslationally modified amino acids residues. Antibodies with binding sites on microtubule surface make it possible to study interaction between microtubules and interacting proteins, including tubulin modifying enzymes, in resting cells or cells activated by extracellular stimuli. Site-directed antibodies can also be used for detection of conformation changes in microtubules due to the presence of flexible tubulin domains [12,13]. In spite of a growing number of available anti-tubulin antibodies, data on location of epitopes on native microtubules outside the $\mathrm{C}$-terminal regions of tubulin subunits are very limited. Comparative (homology) modeling makes it possible to predict the structures of proteins with similar sequences [14]; homology modeling of tubulin subunits was used for calculation of discernible differences in tubulin biophysical properties [15] and for a rational design of plant herbicides [16]. However, plant microtubule models were, so far, not reported.

Previously we have found fixation-dependent exposure of tubulin epitopes in N. tabacum microtubules [17], and phosphorylation of $N$. tabacum tubulin on tyrosine [18]. However, important questions remained unresolved, namely whether or not cellular microtubules can be phosphorylated and what consequences it might have for microtubular integrity. Furthermore, the function of microtubules in cells responding to extracellular stimuli might be better understood with more knowledge on how the predictions of $\beta$-tubulin epitopes and phosphotyrosine locations derived from microtubule model correlate with their exposure in cells.

Here we report on the correlation between localization of small $\beta$-tubulin regions on A. thaliana microtubule protofilament model and their exposure on cortical microtubules in cells.

\section{Results}

\section{Epitope mapping}

Brain tubulin, which can be prepared to a very high degree of purity, is a useful source for epitope mapping. Previous experiments have shown that antibodies TU-14 [19] and TUB 2.1 [20] recognize epitopes in the C-terminal structural domain of porcine brain $\beta$-tubulin, while antibody $18 . \mathrm{D} 6$ recognizes epitope in the $\mathrm{N}$-terminal region of the molecule [21]. It is known that specific chemical proteolysis (by means of $75 \%$ formic acid) of aspartic-proline bonds generates a small number of proteolytic fragments in tubulin dimers [22]. We therefore analysed by immunoblotting porcine brain $\beta$-tubulin fragments after formic acid cleavage, using anti- $\beta$-tubulin antibodies with known epitope location as markers. $\beta$-Tubulin has two aspartic-proline bonds at positions 及31-32 and $\beta 304-305$ (P02554 in the Swiss-Prot Sequence Database) [23]. Formic acid cleavage generates 5 fragments denoted $\beta 1, \beta 2, \beta 3, \beta 1+\beta 2$ and $\beta 1+\beta 3$, as outlined in Figure 1A. The subunits were effectively separated by electrophoresis (Figure 1B, lane 1), and the $\beta$-tubulin subunit was isolated from gel by electroelution (Figure $1 \mathrm{~B}$, lane 2 ). When purified $\beta$-tubulin was subjected to formic acid proteolysis, fragments $\beta 1+\beta 3, \beta 1$ and $\beta 2$ were discernible after staining the blotted proteins with SYPRO Ruby Protein Blot Stain (Figure 1C, lane 1). Antibody 18D6, raised against peptide $\beta 1-12$, served as a marker of the $\beta 1+\beta 3$ fragment (Figure $1 C$, lane 2), antibody TU-06 recognizing an epitope in the region $\beta 81-95$ served as a marker of the $\beta 1$ fragment

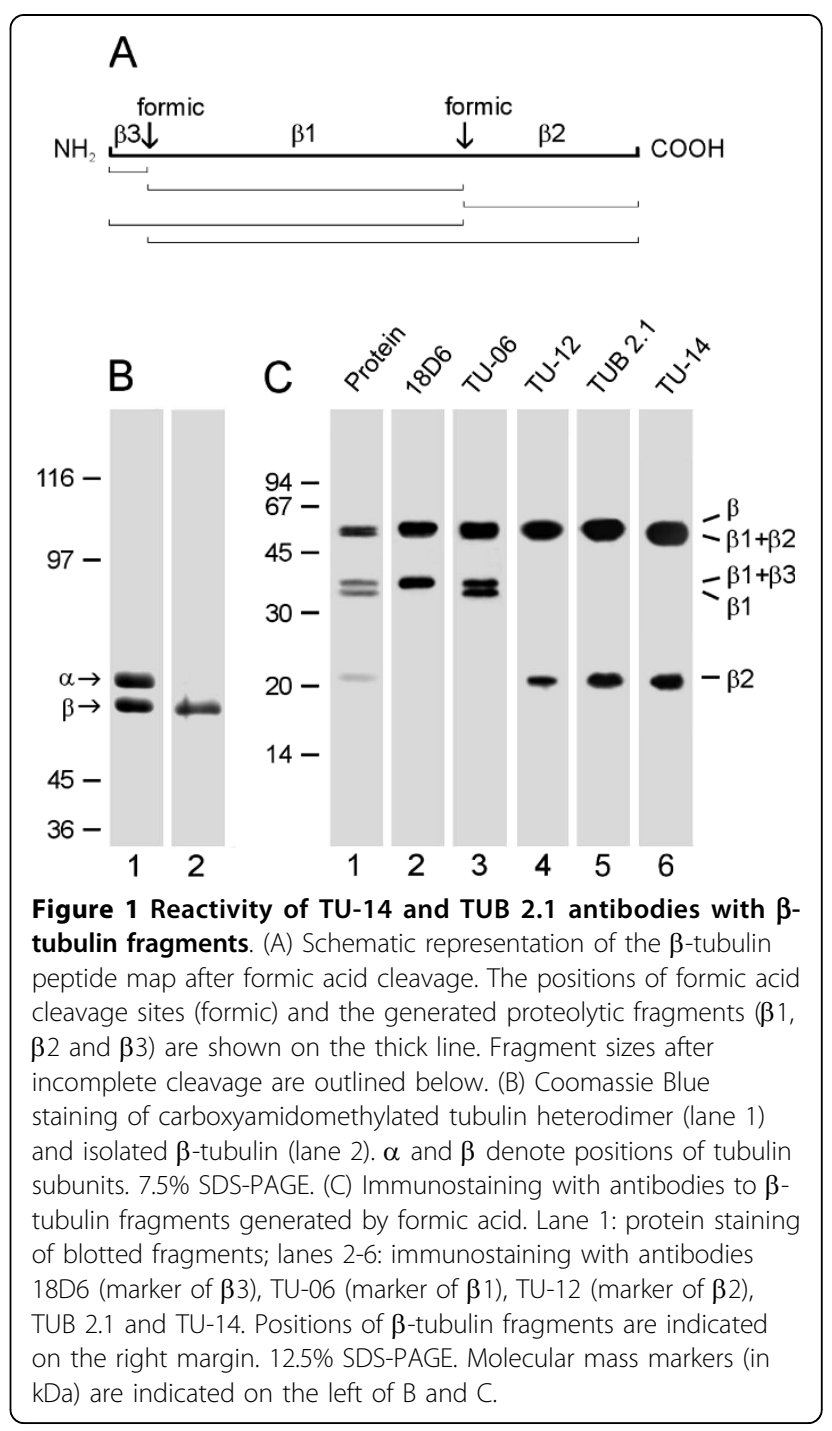


(Figure 1C, lane 3), and antibody $\mathrm{TU}-12$ recognizing an epitope in the region $\beta 426-435$ served as a marker of the $\beta 2$ fragment (Figure $1 C$, lane 4 ). Immunoblotting with TUB 2.1 antibody revealed reactivity with both $\beta 2$ and $\beta 1+\beta 2$ fragments (Figure $1 \mathrm{C}$, lane 5 ). This indicates that the corresponding epitope is located in the region $\beta 305-445$. Antibody TU-14 also reacted with both $\beta 2$ and $\beta 1+\beta 2$ fragments (Figure $1 \mathrm{C}$, lane 6 ).

Peptide scans of immobilized overlapping peptides were used for a more accurate epitope location on A. thaliana $\beta$-tubulin. Two peptide scans were selected covering the regions $\beta 1-180$ (34 linear 15-meric peptides with 5 amino acid overlaps) and $\beta 171-447$ (54 linear 15-meric peptides with 5 amino acid overlaps) in $\beta$-tubulin 1 (TBB1). Results of immunostaining with the antibodies are shown in Figure 2. Using this approach the epitopes were located in the following $\beta$-tubulin regions: $\beta 1-10$ (18D6), $\beta 81-95$ (TU-06), $\beta 426-435$ (TU-12), $\beta 426-435$ (TUB 2.1) and $\beta 436-447$ (TU-14). The comparison of epitope sequences, recognized by antibodies, with corresponding consensus sequences derived from all $\beta$-tubulin isotypes of $A$. thaliana is shown in Table 1 . These data demonstrate that epitopes recognized by antibodies 18D6, TU-06 and TU-12/ TUB 2.1 are highly conserved and are present in all $\beta$ tubulin isotypes. Less conserved is the C-terminal region,

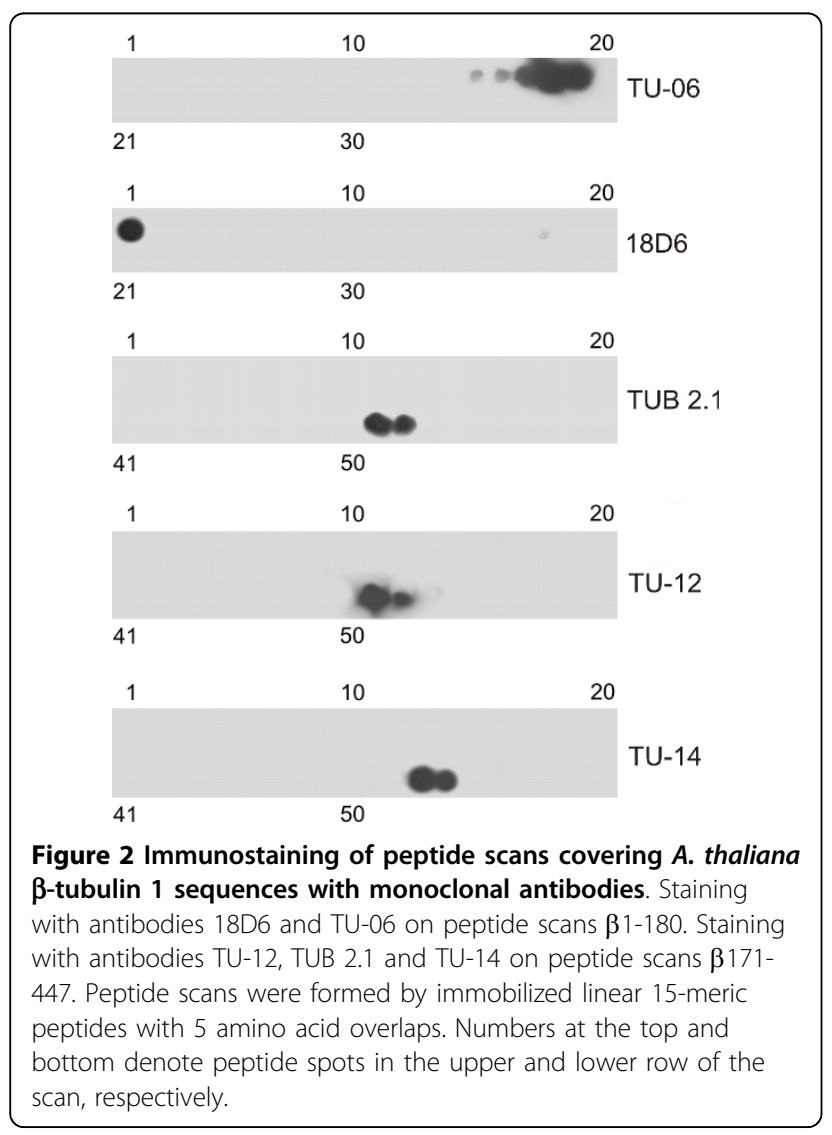

$\beta 436-447$, where the epitope recognized by antibody TU14 is located. Collectively taken, the data demonstrate that the identified $\beta$-tubulin epitopes are conserved and present in A. thaliana $\beta$-tubulins.

\section{Location of $\beta$-tubulin epitopes on a model of $A$. thaliana microtubule protofilaments and in cells}

The position of tubulin dimers in microtubule wall is known for brain tubulin $[8,9]$. Homology modeling of $A$. thaliana microtubules revealed that epitopes 18D6 and TU-06 are exposed to the lumen of microtubules, whereas epitopes for antibodies TU-12/TUB 2.1 and TU-14 are on the external side of microtubules exposed to cytoplasm. Exposures of the epitopes on A. thaliana microtubule protofilaments are depicted in Figure 3. Amino acids that were expected to be exposed on microtubular surface are highlighted in Table 1.

To verify the predicted location of epitopes on microtubules, immunofluorescence experiments were performed on fixed and unfixed detergent-extracted samples prepared from $A$. thaliana seedlings. All tested anti- $\beta$-tubulin antibodies stained microtubules in aldehyde-fixed $A$. thaliana seedlings. The antibodies decorated typical microtubule structures appearing during the cell cycle (interphase microtubules, preprophase band, mitotic spindle and phragmoplast). The antibodies stained microtubules along their whole length, and no differences among individual antibodies were detected. TU-06 failed to stain microtubules on unfixed detergent-extracted cells, in contrast to other antibodies that did stain all microtubule structures. Staining of interphase cortical microtubules in fixed and unfixed A. thaliana epidermal cells of primary roots under

Table 1 Epitope location on A. thaliana $\beta$-tubulins*

\begin{tabular}{|c|c|c|}
\hline Antibody & & Region of epitope location \\
\hline & & 10 \\
\hline \multirow[t]{3}{*}{$18 \mathrm{D} 6$} & TBB1 & M R E I L H V Q G G \\
\hline & Consensus & $\underline{\text { M R E I L H I Q G G }}$ \\
\hline & & 81 \\
\hline \multirow[t]{3}{*}{ TU-06 } & TBB1 & P Y G $Q$ I F R D N F V F Q \\
\hline & Consensus & $\underline{P}$ Y G Q I F R P D F V F G Q \\
\hline & & $\begin{array}{ll}426 & 435\end{array}$ \\
\hline \multirow[t]{3}{*}{ TU-12/TUB 2.1} & TBB1 & Y Q D A T A E E D \\
\hline & Consensus & $\underline{Y Q D A T A D E E ~ G}$ \\
\hline & & 436 \\
\hline \multirow[t]{2}{*}{ TU-14 } & TBB1 & E Y D E E E Q - - V Y E S \\
\hline & Consensus & E Y E E E E E E E \\
\hline
\end{tabular}

* A. thaliana consensus $\beta$-tubulin sequences were generated from $A$. thaliana sequences for TBB1 (P12411), TBB2 (P29512), TBB4 (P24636), TBB5 (P29513), TBB6 (P29514), TBB7 (P29515), TBB8 (P29516) and TBB9 (P29517) using Vector NTI Advance programme (InforMax, Bethesda, MD, USA). Swiss-Prot accession numbers are in parentheses. Identical amino acid positions are underlined. Amino acid residues exposed on inner or outer side of microtubule wall are in bold. 


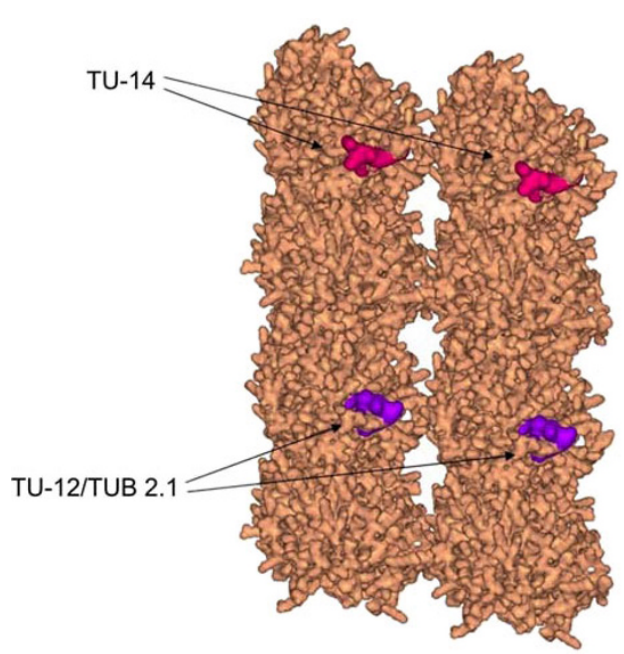

outer side

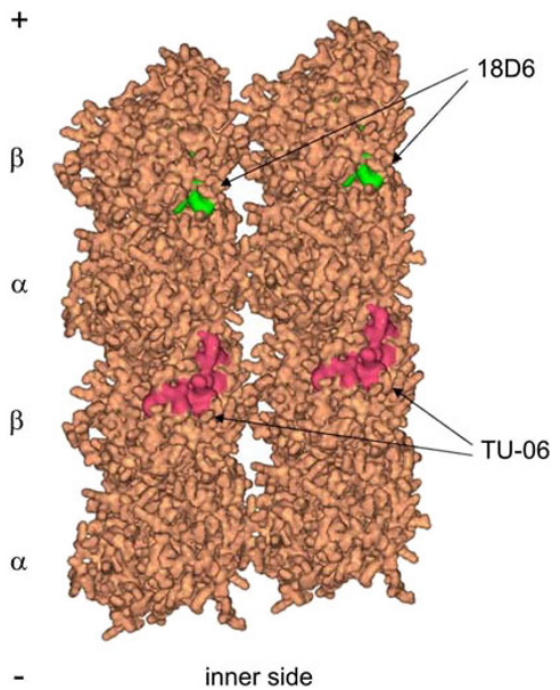

Figure 3 Model of $\boldsymbol{A}$. thaliana microtubule protofilaments with denoted positions of $\boldsymbol{\beta}$-tubulin epitopes. Amino acids exposed on surface of protofilaments are colored for epitopes recognized by monoclonal antibodies 18D6, TU-06, TU-12, TUB 2.1 and TU-14. Outer and inner sides of adjacent protofilaments are depicted. $\alpha$ and $\beta$ denote positions of tubulin subunits in protofilaments; $(+)$ and $(-)$ mark the orientation of protofilaments.

different fixation conditions is demonstrated for antibodies 18D6 and TU-06 (Figure 4A-D). The absence of reactivity with TU-06 could not be attributed to the lack of microtubules, since it was still possible to visualize microtubules by polyclonal anti-tubulin antibody (not shown). The reactivity of antibodies with $A$. thaliana microtubules are summarized in Table 2. These data demonstrate that epitopes TU-12/TUB 2.1 and TU-14,
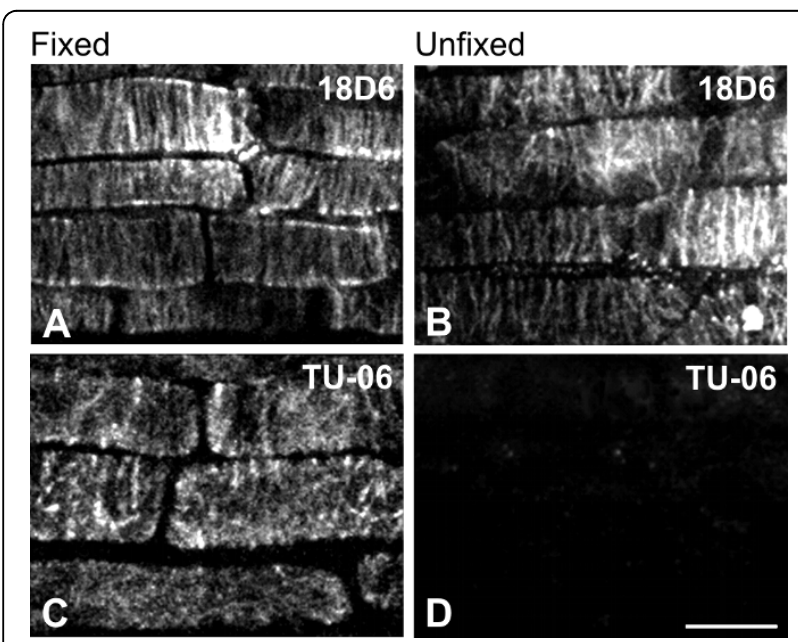

Figure 4 Immunofluorescence staining of $A$. thaliana microtubules with antibodies to $\beta$-tubulin. Fixed $(A, C)$ or unfixed, detergent-extracted $(B, D)$ preparations of primary root epidermal cells were prepared as described in Material and Methods and stained with monoclonal antibodies 18D6 (A-B) and TU-06 (C-D). Bar, $10 \mu \mathrm{m}$. located in the C-terminal structural domain of $\beta$-tubulin and predicted to be on the microtubule surface, are indeed available for antibody staining in unfixed plant cells. On the other hand, TU-06 epitope exposed to the microtubule lumen was not accessible for antibody binding in unfixed microtubules, but fixation made the exposure of that epitope possible. Interestingly, the 18D6 epitope, located on the very N-terminal end of $\beta$-tubulin and also predicted to be exposed towards the microtubular lumen, was available for antibody binding in unfixed preparations.

\section{Exposure of phosphotyrosines on microtubules}

The screening, by means of DS Visualizer program, of tyrosine residues exposed in A. thaliana microtubule model disclosed that only tyrosines 426 and 437 out of the 16 tyrosine residues present in consensus sequence of all $\beta$-tubulin isotypes were completely exposed on the outer side of microtubule surface. Besides, tyrosines at positions $439,444,445,447$ and 451 , present only on

Table 2 Reactivity of anti- $\beta$-tubulin antibodies with A. thaliana microtubules

\begin{tabular}{llll}
\hline Antibodies & Epitope location & \multicolumn{2}{l}{ Microtubules } \\
\cline { 3 - 4 } & & Unfixed & Fixed \\
\hline 18D6 & $1-10$ & + & + \\
TU-06 & $81-95$ & + & - \\
TU-12/TUB2.1 & $426-435$ & + & + \\
TU-14 & $436-447$ & + & + \\
\hline
\end{tabular}


some $\beta$-tubulin isotypes, were found to be part of the flexible C-terminal tail. Thus, they too are exposed on the microtubular external side (Additional file 1).

To test whether or not plant microtubules can carry phosphorylation on tubulin tyrosine residues exposed on the outer side of microtubules, we have performed double-label staining experiments on unfixed detergentextracted $A$. thaliana microtubules using polyclonal anti-phosphotyrosine antibody (P-Tyr) and monoclonal anti- $\beta$-tubulin antibody (TUB 2.1 ). Staining was also performed on seedlings pretreated for $40 \mathrm{~min}$ with 2.5 $\mathrm{mM}$ sodium orthovanadate. This compound is a potent inhibitor of protein tyrosine phosphatases, and proteins are therefore retained in their phosphorylated state. While the signal detected with anti-phosphotyrosine antibody in untreated samples was low (Figure 5C), staining was markedly enhanced if the cells were pretreated with sodium orthovanadate (Figure 5D). Doublelabel staining with anti- $\beta$-tubulin antibody confirmed that microtubules were preserved (Figures $5 \mathrm{~A}$ and $5 \mathrm{~B}$ ), and phosphotyrosine labeling correlated with cortical microtubules in interphase cells (Figure 5F). Similar

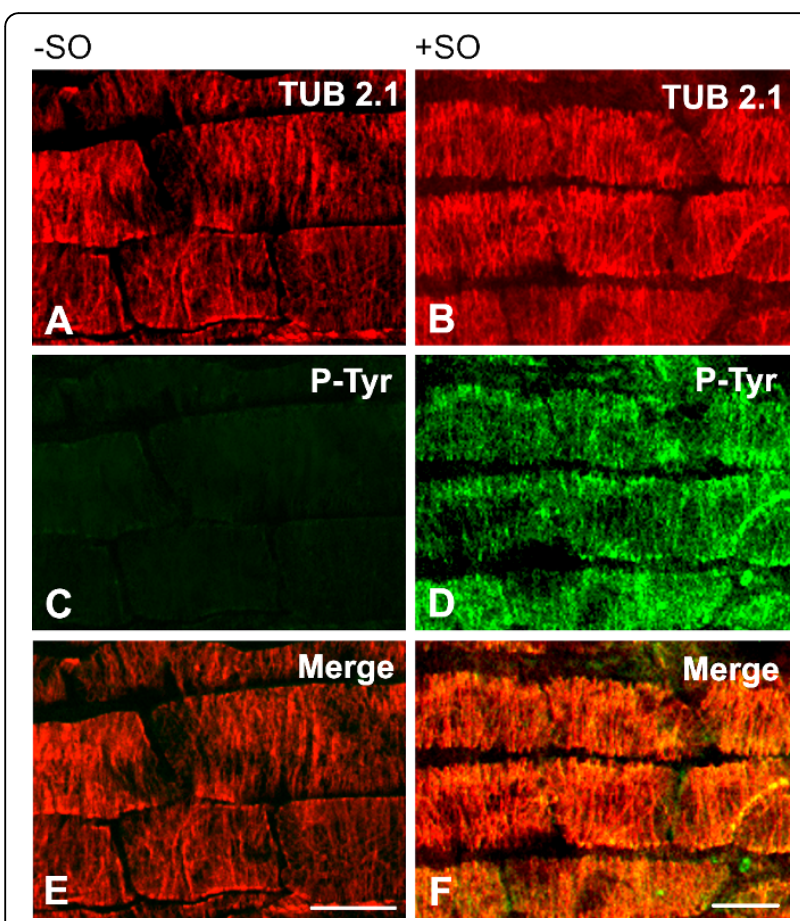

Figure 5 Immunofluorescence double-label staining of A. thaliana unfixed microtubules with antibodies to $\beta$-tubulin and phosphotyrosine. Preparations of primary root epidermal cells, preincubated without $(A, C, E)$ or with $(B, D, F)$ sodium orthovanadate $(\mathrm{SO})$ to inhibit phosphatases were stained with monoclonal antibody TUB 2.1 to $\beta$-tubulin (A-B) and polyclonal antiphosphotyrosine antibody P-Tyr (C-D). Figures A, C, E and B, D, F represent the same field. Superpositions (Merge) are shown in $E$ and F. Bar, $10 \mu \mathrm{m}$. staining pattern with P-Tyr was observed when untreated or sodium orthovanadate-treated samples were fixed before immunostaining. Moreover, clear staining of mitotic spindles was also observed in fixed samples pretreated with sodium orthovanadate (not shown). These data suggest that plant microtubules can be modified by tyrosine phosphorylation and that tyrosine residues located in the $\mathrm{C}$-terminal region of $\beta$-tubulin isotypes, exposed on microtubule surface, could be phosphorylated.

\section{Discussion}

Homology modeling has been used for specification of some biophysical properties of tubulin isotypes [15], as well as for a rational design of microtubule inhibitors [16]. We made use of this approach to predict the exposure of $\beta$-tubulin epitopes and tyrosine residues on the surface of $A$. thaliana microtubules. Here, we report on three novel findings: (1) Identification of phylogenetically conserved $\beta$-tubulin epitopes recognized by wellestablished monoclonal antibodies. (2) Docking of epitopes into A. thaliana microtubule model and confirmation of predicted localization by staining of cellular microtubules. (3) Prediction of tyrosine exposure on the outer side of $A$. thaliana microtubule wall and experimental verification of microtubule phosphorylation on tyrosine in cells.

We confirmed the localization of epitopes for antibo-

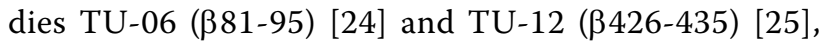
previously determined on porcine brain tubulin, using A. thaliana overlapping $\beta$-tubulin peptides. We improved the accuracy of localization for epitopes recognized by antibodies 18D6 ( $\beta 1-10)$, TUB 2.1 ( $\beta 426-$ $435)$ and TU-14 ( $\beta 436-447)$. Out of this panel, antibodies TU-06, TU-12 and TUB2.1 are commercially available and are supplied by various vendors. Whereas the amino acid sequences in the regions $\beta 1-10$ and $\beta 81-95$ are highly homologous in all eight $\beta$-tubulin isotypes of $A$. thaliana, there are differences in the $\mathrm{C}$-terminal regions of $\beta$-tubulin isotypes demonstrated by their amino acid alignment (Additional file 1). This indicates that the distribution of epitopes recognized by antibodies TU-12, TUB 2.1 and TU-14 might be restricted just to some isotypes. Identification of minimal peptides that bind to the antibodies is necessary before making final conclusions about the distribution of epitopes on individual $\beta$-tubulin isotypes. On the other hand, antibodies 18D6 and TU-06 represent general probes that ought to recognize all $A$. thaliana isotypes. As known posttranslational modifications of $\beta$-tubulin isotypes are located in the C-terminal domains $[5,26]$, modifications should not impair the reactivity of these antibodies. 18D6 and TU-06 could be therefore useful for detection of all $\beta$-tubulin charge variants (isoforms) generated by 
combination of different $\beta$-tubulin isotypes and their posttranslational modifications.

Since the sequence alignment of porcine and A. thaliana $\beta$-tubulin isotypes revealed approximately $85 \%$ sequence identity, it is reasonable to assume that homology modeling based on known structure of bovine brain tubulin [6] combined with the high resolution model of microtubules [8,9] produces a model of $A$. thaliana microtubule protofilaments acceptable with a high degree of confidence. This enabled to position the $\beta$-tubulin epitopes into A. thaliana microtubule wall, and to localize those amino acid residues that are exposed on the inner or outer surface of microtubules.

While decoration of unfixed cellular microtubules was in good agreement with the predicted epitope docking for antibodies TU-06, TU-12, TUB 2.1 and TU-14, decoration of unfixed microtubules with antibody $18 \mathrm{D} 6$ was surprising. The 18D6 epitope in protofilament model was positioned in the microtubule interior facing the lumen, so one would expect that it will be obscured on unfixed microtubules. In addition, it has been demonstrated previously that microinjected 18D6 antibody did not decorate microtubules in animal $\mathrm{CHO}-\mathrm{K} 1$ cells, if the cells were prior to fixation extracted with $0.5 \%$ Triton X-100 in microtubule stabilizing buffer [21]. When taxol was excluded during preparation of unfixed samples, staining of microtubules with 18D6 antibody was reduced (Additional file 2). An altering effect of taxol on the arrangement of tubulin protofilaments was reported $[27,28]$. We thus propose that the exposure of 18D6 epitope on unfixed $A$. thaliana microtubules might be caused by conformation changes generated by taxol used for stabilization of microtubules. Interestingly, only three out of the ten amino acids of the 18D6 epitope are exposed on the interior surface of microtubules (Table 1); this indicates that the $\mathrm{N}$-terminal $\beta$-tubulin region is important for antibody binding to microtubules.

On the other hand, finding that epitopes 18D6 and TU-06, located on the inner site of microtubule wall, appeared after fixation of microtubules was no surprise. It is well documented that different fixation conditions influence the exposure of tubulin epitopes both in animal [29] and plant microtubules [17]. Since tubulin subunits have flexible domains [30], even "mild" fixation with $0.2 \%$ formaldehyde can affect the exposure of epitopes on microtubules [13]. It was suggested that changes in the conformation of tubulin by fixation influence the interaction between protofilaments, and can consequently lead to an exposure of epitopes in the lumen of microtubules, as was demonstrated for acetylated lysine $\alpha 40$ [13]. Conformation changes in protofilaments were reported after binding of the motor proteins to microtubules $[31,32]$. Our results strengthen the hypothesis that the way samples are prepared can substantially influence the results obtained in cells, and a good deal of caution must be used when predicting locations inferred from microtubule models.

Previously we have shown that plant tubulin is phosphorylated on tyrosine [18]; however, data on tyrosine phosphorylation of microtubules in plant cells are missing. Homology modeling in A. thaliana revealed that tyrosines in the $\mathrm{C}$-terminal region of $\beta$-tubulin isotypes (behind $\beta 425$ ) are exposed on the microtubule outer side and might therefore become likely targets for tyrosine kinases. In the case of $\alpha$-tubulin isotypes, homology modeling disclosed that out of the total of 17 tyrosine residues only the $\mathrm{C}$-terminal tyrosine $\alpha 450$ was completely exposed on the surface of microtubules (Additional file 1). To verify that tyrosines on polymerized microtubules are indeed phosphorylated, immunofluorescence experiments were carried out with unfixed cells. The results showed that the anti-phosphotyrosine antibody decorated microtubules. While the steady-state level of microtubule phosphorylation on tyrosine was low, phosphorylation of microtubules was clearly visible in cells pretreated with sodium orthovanadate, a factor which shifts the equilibrium to the phosphorylation state. Although one cannot exclude that the tyrosines partly buried in the microtubule wall are exposed to phosphorylation after binding of kinases to microtubules, it seems reasonable to assume that phosphorylation on tyrosines could occur in the C-terminal regions of polymerized tubulins. Since immunoblotting after 2D-PAGE separation of isolated N. tabacum tubulin clearly confirmed phosphorylation of both subunits [18], we suggest that $\beta$-tubulin as well as $\alpha$-tubulin isotypes in A. thaliana microtubules might be phosphorylated. MAPs could also be phosphorylated on tyrosine in sodium orthovanadate-treated cells. However, MAPs are less abundant than tubulin in microtubule preparations isolated from plant extracts, and show usually punctuate localization in cortical A. thaliana microtubules [33]. In contrast, we have observed a uniform staining along the microtubules with anti-phosphotyrosine antibody in fixed cells. This indicates that anti-phosphotyrosine antibody might stain tubulin. However, further studies are necessary to confirm this assumption.

It is well established that the C-terminal domains of tubulins in polymerized microtubules can be posttranslationally modified [26] and bind MAPs [2]. The physiological significance of tyrosine phosphorylation of microtubules is unknown, but it can possibly modulate the binding of MAPs or motor proteins. In addition, it might modulate the function of microtubules in response to extracellular stimuli. Cellular microtubules can provide a matrix upon which the signaling molecules are arrayed [34]. Tyrosine phosphorylation of tubulin in microtubule polymer might affect the cell 
signaling by enhancing the Src-homology 2 (SH2) domain-mediated interaction between microtubules and signaling molecules. Syk-dependent phosphorylation of microtubules during activation of B-lymphocytes was described [35]. The Syk kinase belongs to protein tyrosine kinases of Syk/ZAP family, and tubulin is one of the proteins in animal cells identified as a likely substrate for Syk in vivo [36]. It is worth mentioning that ZAP-like kinases were identified by bioinformatics homology analysis in plant cells (Y.B. Blume unpublished result). Recent experiments with tyrosine kinase and phosphatase inhibitors identified tyrosine phosphorylation as an important factor in overall organization of microtubules in A. thaliana root cells [37].

\section{Conclusions}

Using homology modeling we have generated an Arabidopsis thaliana microtubule protofilament model that served for the prediction of surface exposure of five $\beta$ tubulin epitopes, as well as tyrosine residues. Peptide scans newly disclosed the position of epitopes detected by antibodies 18D6 ( $\beta 1-10)$, TUB2.1 ( $\beta 426-435)$ and TU-14 ( $\beta 436-445)$. Experimental verification of obtained results by immunofluorescence microscopy revealed that the exposure of epitopes was dependent on fixation. Moreover, homology modeling showed that only tyrosines located in the $C$-terminal region of $\beta$-tubulins (behind $\beta 425$ ) are exposed on the microtubule outer side. Immunofluorescence microscopy confirmed tyrosine phosphorylation of microtubules in plant cells, and $\beta$-tubulins could thus be one of the targets for tyrosine kinases. Tyrosine phosphorylation of microtubules may affect their capability to interact with associated proteins in cells responding to extracellular stimuli. The data also demonstrate that however useful the homology modeling may be for the prediction of amino acid exposure on microtubule surface, it has to be corroborated by experimental verification.

\section{Methods}

\section{Antibodies}

The following mouse monoclonal antibodies to $\beta$-tubulin were used: TU-12 (IgM) recognizes an epitope located in the $\beta 426-435$ region [25] of the C-terminal structural domain [38]; TU-14 (IgM) recognizes an epitope located in the isotype-defining region [19] of the C-terminal structural domain; TU-06 (IgM) recognizes an epitope located in the $\beta 81-95$ region [24] of the Nterminal structural domain of $\beta$-tubulin [29]; TUB 2.1 (IgG1) (Sigma, Prague, Czech Republic) recognizes an epitope located in the C-terminal structural domain of $\beta$-tubulin [20]; 18D6 (IgG1) was raised against the $\mathrm{N}$ terminal peptide of $\beta$-tubulin ( $\beta 1-12)$ [21]. Absence of any cross-reactivity with $\alpha$-tubulin has previously been confirmed for all anti- $\beta$-tubulin antibodies used in this study $[17,21,39]$. Microtubules were also visualized by affinity purified rabbit antibody to tubulin [38]. Mouse monoclonal antibody VI-01 (IgM) to vertebrate vimentin [40] was used as a negative control. Rabbit antibody to phosphotyrosine was from Upstate Biotechnology (Lake Placid, NY, USA). Anti-mouse antibody conjugated with horseradish peroxidase was purchased from Promega Biotech (Madison, WI, USA). Anti-mouse antibodies conjugated with fluorescein isothiocyanate (FITC) or indocarbocyanate (Cy3), and anti-rabbit antibody conjugated with FITC were obtained from Jackson Immunoresearch Laboratories (West Grove, PA, USA).

\section{Cells}

Seedlings of Arabidopsis thaliana (Columbia 0 ecotype) were grown under sterile conditions on a half strength Murashige and Skoog (MS) medium containing vitamins (Duchefa, Haarlem, Netherlands), supplemented with 10 $\mathrm{g} / \mathrm{L}$ sucrose and solidified with $4 \mathrm{~g} / \mathrm{L}$ Gelrite (Duchefa) at $\mathrm{pH}$ 5.7. After overnight incubation at $4^{\circ} \mathrm{C}$, the dishes were placed vertically in a growth chamber at $22^{\circ} \mathrm{C}$ in a 16/8 h (light/dark) photoperiod. Four-day-old seedlings were used for experiments.

\section{Protein preparation and epitope mapping}

Microtubule protein from porcine brain was prepared by three temperature-dependent cycles of assembly and disassembly according to Shelanski [41]. Tubulin free of microtubule-associated proteins was obtained by phosphocellulose chromatography [42], and was stored in small aliquots in liquid nitrogen. To separate $\alpha$ - and $\beta$ tubulin subunits, tubulin was carboxyamidomethylated and the subunits were effectively separated by SDSPAGE according to Laemmli using modifications in separation gel and electrode buffer composition [43]. Subunits were isolated from the gel by electroelution using Model 422 Electro-Eluter (Bio-Rad Laboratories, Richmond, CA) according to manufacturer's directions. Electroelution was performed in $50 \mathrm{mM}$ ammonium bicarbonate containing $0.1 \%$ SDS. Eluted proteins were concentrated in Speed Vac (SAVANT Instruments, Farmingdale, NY).

Chemical proteolysis of $\beta$-tubulin was performed by dissolving the isolated protein in $75 \%$ formic acid and incubating the solution in the dark at $37^{\circ} \mathrm{C}$ for $24 \mathrm{~h}$ [22]. After dialysis against water using a dialysis membrane with MWCO 3,500 Daltons, samples were mixed with five-times concentrated SDS-sample buffer and analyzed by immunoblotting.

For epitope mapping, the synthetic overlapping peptides (15-meric peptides with 5 amino acid overlaps) were prepared by SPOT synthesis (Jerini Peptide Technologies, Berlin, Germany). Each spot carried 
approximately 5 -nmol peptide covalently bound to the cellulose- $\beta$-alanine membrane. Peptide scans covered the sequences $\beta 1-180$ in the $\mathrm{N}$-terminal structural domain (34 peptides) and $\beta 171-447$ in the C-terminal structural domain (53 peptides) of $A$. thaliana $\beta$-tubulin 1 (TBB1, accession number P12411 in the Swiss-Prot Sequence Database). Epitope mapping was performed according to the manufacturer's directions with chemiluminescent detection of bound antibodies.

\section{Preparation of cytoskeletons}

Fixation of $A$. thaliana seedlings was adapted from Le et al. [44]. All incubation and washing steps were performed at room temperature. Briefly, whole seedlings of $A$. thaliana were fixed for $2 \mathrm{~h}$ in a freshly prepared mixture of $4 \%(\mathrm{w} / \mathrm{v})$ paraformaldehyde and $0.5 \%(\mathrm{v} / \mathrm{v})$ glutaraldehyde in PHEM buffer (60 mM PIPES-KOH, pH 7.0, containing 25 mM HEPES, 2 mM EGTA, 2 $\mathrm{mM} \mathrm{MgSO}_{4}$ ) supplemented with $0.05 \%(\mathrm{v} / \mathrm{v})$ Triton X100 and $0.2 \mathrm{mM}$ phenylmethane sulfonyl fluoride (PMSF). After rinsing in PHEM the seedlings were incubated in $0.1 \%(\mathrm{w} / \mathrm{v}) \mathrm{NaBH}_{4}$ in PHEM for $15 \mathrm{~min}$ to reduce free aldehydes. Cell wall was digested for 20 min with an enzyme cocktail containing $0.1 \%(\mathrm{v} / \mathrm{v})$ pectinase (Sigma) and $0.05 \%(\mathrm{w} / \mathrm{v})$ pectolyase (Sigma) in PHEM. Seedlings were washed in PBS and incubated for $20 \mathrm{~min}$ in $1 \%(\mathrm{w} / \mathrm{v}) \mathrm{BSA}$ in PBS to prevent unspecific binding of antibodies.

To prepare unfixed samples, $A$. thaliana seedlings were treated with the enzyme cocktail, washed twice for $10 \mathrm{~min}$ in $0.4 \mathrm{M}$ glycerol in PHEM (glycerol/PHEM) and incubated for $15 \mathrm{~min}$ in the same buffer containing $1 \%(\mathrm{v} / \mathrm{v})$ Triton X-100, $0.2 \mathrm{mM}$ PMSF and $10 \mu \mathrm{M}$ taxol (Sigma). In some cases, taxol was omitted from incubation buffer during preparation of unfixed samples. After washing in glycerol/PHEM, the cells were used for immunostaining.

For some experiments, seedlings were pretreated for $40 \mathrm{~min}$ at room temperature with $2.5 \mathrm{mM}$ sodium orthovanadate (Sigma) before preparation of fixed or unfixed samples, in order to inhibit tyrosine phosphatases. $250 \mathrm{mM}$ sodium orthovanadate stock solution in $\mathrm{H}_{2} \mathrm{O}$ was prepared immediately before use [37].

\section{Immunofluorescence microscopy}

All antibody dilutions were made with $0.5 \%(\mathrm{w} / \mathrm{v}) \mathrm{BSA}$ in PBS supplemented with $2 \mu \mathrm{M}$ leupeptin (Sigma). Antibodies TU-06, TU-12 and TU-14 in the form of culture supernatants were diluted 1:50, antibodies TUB 2.1 and 18D6 were diluted 1:100. Fixed samples were incubated with primary antibodies for $2.5 \mathrm{~h}$, washed 3 times for $5 \mathrm{~min}$ in PBS and incubated for $1.5 \mathrm{~h}$ with FITC-conjugated anti-mouse antibodies diluted 1:30. $A$. thaliana seedlings were incubated with antibodies in suspension and transferred before examination on glass coverslips with a small drop of PBS.

For experiments with unfixed cytoskeletons, seedlings of $A$. thaliana were incubated for $2.5 \mathrm{~h}$ with primary antibodies diluted in glycerol/PHEM. After washing off the unbound antibodies by glycerol/PHEM, samples were fixed for $1.5 \mathrm{~h}$ with $4 \%(\mathrm{w} / \mathrm{v})$ paraformaldehyde in glycerol/PHEM. Samples were then washed with PHEM without glycerol and incubated for $1.5 \mathrm{~h}$ with secondary antibody as described above, followed by transfer on glass coverslips.

For double-label immunofluorescence examination of fixed or unfixed samples of $A$. thaliana seedlings, the rabbit anti-phosphotyrosine antibody P-Tyr (1:100) and the mouse monoclonal antibody TUB 2.1 (1:100) were applied simultaneously. FITC-conjugated anti-rabbit and Cy3-conjugated anti-mouse antibodies were diluted 1:100 and 1:500, respectively.

Preparations of epidermis of $A$. thaliana primary root were examined with confocal laser scanning microscope LSM 510 META (Carl Zeiss, Jena, Germany) with a Plan-Apochromat $63 \times$ oil-immersion objective. FITC emission was excited using the 488-nm ray of the argon laser, whereas Cy3 was exited using the 543-nm line of the helium/neon laser. Emission signals of FITC and Cy3 were separated by means of META system with, respectively, BP 505-530 and LP 560 filter.

\section{Gel electrophoresis and immunoblotting}

Details on the one-dimensional SDS-polyacrylamide gel electrophoresis (SDS-PAGE), electrophoretic transfer of separated proteins onto nitrocellulose, and immunostaining procedure are described elsewhere [45]. SYPRO Ruby Protein Blot Stain (Invitrogen, Carlsbad, CA, USA) was used for the detection of proteins on nitrocellulose. The antibodies TU-06, TU-12 and TU14 in the form of culture supernatants were diluted $1: 5$, TUB 2.1 and 18D6 were diluted, respectively, $1: 2,000$ and $1: 10,000$. Peroxidase-conjugated antibody was diluted 1:10,000. Bound antibodies were detected by SuperSignal WestPico Chemiluminescent reagents according to manufacturer's directions (Pierce, Rockford, IL, USA).

\section{Microtubule protofilament modeling}

The spatial structure of $\alpha \beta$-tubulin heterodimers were reconstructed by the homology modeling method [46] using a previously described procedure [47]. For spatial model development, $A$. thaliana amino acid sequences corresponding to tubulin alpha-1 chain (P11139; SwissProt Sequence Database) and tubulin beta-1 chain (P12411; Swiss-Prot Sequence Database) were applied. Preliminary geometry optimization of dimer spatial structure was calculated by the L-BFGS method [48] 
using the mdrun module of GROMACS software [49]. Spatial organization of tubulin protofilaments was computed using serial translation of the heterodimer along microtubule axis on a distance of $79.4 \AA$. Relative arrangement of protofilaments in microtubule was reconstructed by serial geometric transformations including protofilament axial rotation by 14 degrees and protofilament shift angularly of 10 degrees on a distance of $53 \AA$. Spatial structures of calculated longitudinal and lateral contact interfaces were refined to known data about amino acid residues which can be involved in forming the appropriate contacts $[8,9]$. Data visualization and analysis of spatial localization of antibody epitopes were done using the DS Visualizer 2.0 software (Accelrys Software Inc., Cambridge, United Kingdom).

Additional file 1: Supplementary Table 1S. Alignment of carboxy-

terminal domains of $\beta$ - and $\alpha$-tubulins in A. thaliana.

Click here for file

[http://www.biomedcentral.com/content/supplementary/1471-2229-10-

29-S1.PDF ]

Additional file 2: Supplementary Figure 1S. Effect of taxol on

immunofluorescence staining of $A$. thaliana unfixed microtubules.

Click here for file

[http://www.biomedcentral.com/content/supplementary/1471-2229-1029-S2.PDF ]

\section{Acknowledgements}

We thank Dr. DW Cleveland (Ludwig Institute for Cancer Research, University of California at San Diego, La Jolla, USA) for providing antibody 18D6, and V. Richterová for help with documentation. The work was funded partially by INTAS grant 03-51-6459 for AY, YB, PD and VS. The work of TS, VS and PD was also supported from project LC545 (Ministry of Education, Youth and Sport of the (zech Republic) grant No. KAN200520701 from GA ASCR, and by Institutional Research Support AVOZ 50520514.

\section{Author details}

'Institute of Food Biotechnology and Genomics, National Academy of Sciences of Ukraine, Kiev 04123, Ukraine. ${ }^{2}$ Institute of Molecular Genetics, Academy of Sciences of the Czech Republic, 14220 Prague, Czech Republic.

\section{Authors' contributions}

YB conceived the study, participated in homology modeling and helped in drafting the manuscript. AY performed the immunofluorescence experiments with inhibitors and critically evaluated the obtained data. YS prepared the cytoskeletons and participated in confocal microscopy. AN generated the microtubule protofilament model. TS carried out the epitope mapping and immunoblotting experiments. VS isolated the microtubule proteins and tubulins. PD participated in the design and coordination of this study, prepared the tubulin fragments and drafted the manuscript. All authors read and approved the final version of the manuscript.

Received: 23 November 2009

Accepted: 18 February 2010 Published: 18 February 2010

\section{References}

1. Goddard RH, Wick SM, Silflow CD, Snustad DP: Microtubule components of plant cell cytoskeleton. Plant Physiol 1994, 104:1-6.

2. Amos LA, Schlieper D: Microtubules and maps. Adv Protein Chem 2005, 71:257-298.

3. Kopczak SD, Haas NA, Hussey PJ, Silflow CD, Snustad DP: The small genome of Arabidopsis contains at least six expressed alpha-tubulin genes. Plant Cell 1992, 4:539-547.
4. Snustad DP, Haas NA, Kopczak SD, Silflow CD: The small genome of Arabidopsis contains at least nine expressed beta-tubulin genes. Plant Cell 1992, 4:549-556.

5. Verhey KJ, Gaertig J: The tubulin code. Cell Cycle 2007, 6:2152-2160.

6. Nogales E, Wolf SG, Downing KH: Structure of the $\alpha \beta$ tubulin dimer by electron crystallography. Nature 1998, 391:199-203.

7. Löwe J, Li H, Downing KH, Nogales E: Refined structure of $\alpha \beta$-tubulin at 3.5 A resolution. J Mol Biol 2001, 313:1045-1057.

8. Nogales $\mathrm{E}$, Whittaker M, Milligan RA, Downing KH: High-resolution model of the microtubule. Cell 1999, 96:79-88.

9. Meurer-Grob P, Kasparian J, Wade RH: Microtubule structure at improved resolution. Biochemistry 2001, 40:8000-8008.

10. Li H, DeRosier DJ, Nicholson WW, Nogales E, Downing KH: Microtubule structure at 8 A resolution. Structure 2002, 10:1317-1328.

11. Downing KH: Structural basis for the interaction of tubulin with proteins and drugs that affect microtubule dynamics. Annu Rev Cell Dev Biol 2000, 16:89-111.

12. Andreu JM, de Pereda JM: Site-directed antibodies to tubulin. Cell Motil Cytoskel 1993, 26:1-6.

13. Dráberová E, Viklický V, Dráber P: Exposure of lumenal microtubule sites after mild fixation. Eur J Cell Biol 2000, 79:982-985.

14. Chothia C, Lesk AM: The relation between the divergence of sequence and structure in proteins. EMBO J 1986, 5:823-826.

15. Carpenter EJ, Huzil JT, Ludueña RF, Tuszynski JA: Homology modeling of tubulin: influence predictions for microtubule's biophysical properties. Eur Biophys I Biophys Lett 2006, 36:35-43.

16. Tuszynski JA, Huzil JT, Carpenter EJ: Tubulin isotypes and their role in microtubule dynamic instability, implication for modeling and rational design of inhibitors. The plant cytoskeleton: a key tool for agrobiotechnology Dordrecht, The Netherlands: SpringerBlume Y, Baird WV Yemets Al, Breviario D 2008, 305-326.

17. Smertenko $A$, Blume $Y$, Viklický $V$, Dráber P: Exposure of tubulin structural domains in Nicotiana tabacum microtubules probed by monoclonal antibodies. Eur J Cell Biol 1997, 72:104-112.

18. Blume Y, Yemets A, Sulimenko V, Sulimenko T, Chan J, Lloyd C, Dráber P: Tyrosine phosphorylation of plant tubulin. Planta 2008, 229:143-150.

19. Linhartová I, Dráber P, Dráberová E, Viklický V: Immunological discrimination of $\beta$-tubulin isoforms in developing mouse brain. Posttranslational modification of non-class III $\beta$-tubulins. Biochem J 1992, 288:919-924.

20. Matthes T, Wolff A, Soubiran P, Gros F, Dighiero G: Antitubulin antibodies. 2. Natural autoantibodies and induced antibodies recognize different epitopes on the tubulin molecule. J Immunol 1988, 141:3135-3141.

21. Theodorakis NG, Cleveland DW: Physical evidence for cotranslational regulation of beta-tubulin mRNA degradation. Mol Cell Biol 1992, 12:791-799.

22. Serrano L, Wandosell F, Delatorre J, Avila J: Proteolytic modification of tubulin. Methods Enzymol 1986, 134:179-190.

23. Krauhs E, Little M, Kempf T, Hofer-Warbinek R, Ade W, Ponstingl H: Complete amino acid sequence of $\beta$-tubulin from porcine brain. Proc Natl Acad Sci USA 1981, 78:4156-4160

24. Libusová L, Sulimenko T, Sulimenko V, Janisch R, Hozák P, Dráber P: Distinct localization of a beta-tubulin epitope in the Tetrahymena thermophila and Paramecium caudatum cortex. Protoplasma 2005, 225:157-167.

25. Peknicova J, Pexidrova M, Kubatova A, Koubek P, Tepla O, Sulimenko T, Draber P: Expression of beta-tubulin epitope in human sperm with pathological spermiogram. Fertil Steril 2007, 88:1120-1128.

26. Westermann S, Weber K: Post-translational modifications regulate microtubule function. Nat Rev Mol Cell Biol 2003, 4:938-945.

27. Diaz JF, Valpuesta JM, Chacon P, Diakun G, Andreu JM: Changes in microtubule protofilament number induced by Taxol binding to an easily accessible site. Internal microtubule dynamics. J Biol Chem 1998, 273:33803-33810.

28. Mitra A, Sept D: Taxol allosterically alters the dynamics of the tubulin dimer and increases the flexibility of microtubules. Biophys J 2008, 95:3252-3258.

29. Dráber P, Dráberová E, Linhartová I, Viklický V: Differences in the exposure of $\mathrm{C}$ - and $\mathrm{N}$-terminal tubulin domains in cytoplasmic microtubules detected with domain-specific monoclonal antibodies. J Cell Sci 1989, 92:519-528.

30. de Pereda JM, Andreu JM: Mapping surface sequences of the tubulin dimer and taxol-induced microtubules with limited proteolysis. Biochemistry 1996, 35:14184-14202. 
31. Hoenger A, Milligan RA: Motor domains of kinesin and ncd interact with microtubule protofilaments with the same binding geometry. J Mol Biol 1997, 265:553-564.

32. Wendt T, Karabay A, Krebs A, Gross H, Walker R, Hoenger A: A structural analysis of the interaction between ncd tail and tubulin protofilaments. J Mol Biol 2003, 333:541-552.

33. Sedbrook JC, Kaloriti D: Microtubules, MAPs and plant directional cell expansion. Trends Plant Sci 2008, 13:303-310.

34. Gundersen GG, Cook TA: Microtubules and signal transduction. Curr Opin Cell Biol 1999, 11:81-94.

35. Faruki S, Geahlen RL, Asai DJ: Syk-dependent phosphorylation of microtubules in activated B-lymphocytes. J Cell Sci 2000, 113:2557-2565.

36. Peters JD, Furlong MT, Asai DJ, Harrison ML, Geahlen RL: Syk, activated by cross-linking the B-cell antigen receptor, localizes to the cytosol where it interacts with and phosphorylates $\alpha$-tubulin on tyrosine. $J$ Biol Chem 1996, 271:4755-4762.

37. Yemets A, Sheremet Y, Vissenberg K, Van Orden J, Verbelen JP, Blume YB: Effects of tyrosine kinase and phosphatase inhibitors on microtubules in Arabidopsis root cells. Cell Biol Int 2008, 32:630-637.

38. Dráber P, Dráberová E, Viklický V: Immunostaining of human spermatozoa with tubulin domain-specific monoclonal antibodies. Histochemistry 1991, 195:519-524.

39. Gozes I, Barnstable CJ: Monoclonal antibodies that recognize discrete forms of tubulin. Proc Natl Acad Sci USA 1982, 79:2579-2583.

40. Dráberová $E$, Dráber $P$, Havlíèek F, Viklický V: A common antigenic determinant of vimentin and desmin defined by monoclonal antibody. Folia Biol (Prague) 1986, 32:295-303.

41. Shelanski ML, Gaskin F, Cantor CR: Microtubule assembly in the absence of added nucleotides. Proc Natl Acad Sci USA 1973, 70:765-768.

42. Weingarten MD, Lockwood AH, Hwo SY, Kirschner MW: A protein factor essential for microtubule assembly. Proc Natl Acad Sci USA 1975, 72:1858-1868.

43. Sheir-Neiss G, Lai MH, Morris NR: Identification of a gene for beta-tubulin in Aspergillus nidulans. Cell 1978, 15:639-647.

44. Le J, Vandenbussche F, Van Der Straeten D, Verbelen JP: In the early response of Arabidopsis roots to ethylene, cell elongation is up- and down-regulated and uncoupled from differentiation. Plant Physiol 2001, 125:519-522.

45. Dráberová E, Dráber P: A microtubule-interacting protein involved in coalignment of vimentin intermediate filaments with microtubules. J Cell Sci 1993, 106:1263-1273.

46. Krieger E, Nabuurs SB, Vriend G: Homology modeling. Structural Bioinformatics Chichester, UK: John Wiley \& SonsBourne PE, Weissig H 2003, 509-524.

47. Nyporko AY, Blume Y: Comparative analysis of secondary structure of tubulins and FtsZ proteins. Biopolymers and Cell 2001, 17:61-69, (in Russian)..

48. Das B, Meirovitch H, Navon IM: Performance of hybrid methods for largescale unconstrained optimization as applied to models of proteins. $J$ Comput Chem 2003, 24:1222-1231.

49. Lindahl E, Hess B, van der Spoel D: Gromacs 3.0: A package for molecular simulation and trajectory analysis. J Mol Model 2001, 7:306-317.

\section{doi:10.1186/1471-2229-10-29}

Cite this article as: Blume et al: Exposure of beta-tubulin regions defined by antibodies on an Arabidopsis thaliana microtubule protofilament model and in the cells. BMC Plant Biology 2010 10:29.

\section{Submit your next manuscript to BioMed Central and take full advantage of:}

- Convenient online submission

- Thorough peer review

- No space constraints or color figure charges

- Immediate publication on acceptance

- Inclusion in PubMed, CAS, Scopus and Google Scholar

- Research which is freely available for redistribution

Submit your manuscript at www.biomedcentral.com/submit 